\title{
Urban Expansion Assessment in Huaihe River Basin, China, from 1998 to 2013 Using Remote Sensing Data
}

\author{
Yong Fan, ${ }^{1,2}$ Xi Zhu, ${ }^{3}$ Zongyi He, ${ }^{2}$ Shengdi Zhang, ${ }^{4}$ Junbo Gao, ${ }^{1}$ Feiyan Chen, ${ }^{2}$ \\ Xiaolin Peng, ${ }^{2}$ and Junli $\mathrm{Li}^{5}$ \\ ${ }^{1}$ School of Geographic Sciences, Xinyang Normal University, No. 237 Nanhu Road, Xinyang 464000, China \\ ${ }^{2}$ School of Resource and Environmental Sciences, Wuhan University, No. 129 Luoyu Road, Wuhan 430079, China \\ ${ }^{3}$ Shanghai Municipal Institute of Surveying and Mapping, No. 419 Wuning Road, Shanghai 200063, China \\ ${ }^{4}$ School of Geo-Sciences, The University of Edinburgh, Central Campus, Drummond Street, Edinburgh EH89XP, UK \\ ${ }^{5}$ School of Resources and Environment, Anhui Agricultural University, No. 130 Changjiang West Road, Hefei 230036, China
}

Correspondence should be addressed to Zongyi He; zongyihe@tom.com

Received 13 October 2016; Revised 26 February 2017; Accepted 22 March 2017; Published 13 April 2017

Academic Editor: Eduard Llobet

Copyright (C) 2017 Yong Fan et al. This is an open access article distributed under the Creative Commons Attribution License, which permits unrestricted use, distribution, and reproduction in any medium, provided the original work is properly cited.

\begin{abstract}
Urbanization reflects the overall behavior of human society; thus, characterization of its associated spatial and temporal trends has been extensively researched. This study examines the process of urban expansion in the Huaihe River Basin (HRB) which is a key transition region within China's urban system. In order to grasp the urban expansion process in different temporal sequences objectively, rapidly, and accurately, we used remote sensing data to assess the urban expansion in time and space. Urban expansion rules were defined for the urban area, urbanization intensification, extended dynamic degree, and spatial pattern. The research findings show that the urban area expansion speed was at medium level throughout the entire HRB and within each province. Presently, the formation of a whole urban agglomeration or urban system is not complete in the HRB; urban expansion in the HRB displayed space-time disequilibrium tendencies during 1998-2013.
\end{abstract}

\section{Introduction}

Urbanization is an important spatial expression of the human social development process [1]. The similarities and differences between different types of urban expansion can be analyzed by reconstructing the urban expansion process, thereby revealing the inner and outer driving mechanisms in the emergence and development of urban land [2]. In 2008, for the first time in history, the world reached an important milestone: half of the world population was shown to live in urban areas. Moreover, most of the urban population growth has been occurring in developing countries. The space occupied by urban areas is increasing faster than the urban population itself. Between 2000 and 2030, the world's urban population is expected to increase by $72 \%$, and the built-up areas of cities of 100,000 people or more could increase by $175 \%$ [3]. Dramatic urban expansion has occurred universally in developing countries in recent decades [4], and problems such as environmental contamination and urban flooding have consequently emerged [5]. The Huaihe River Basin (HRB) in China represents a transition region between the north and south areas of China's urban system and has a large population, rich resources, and relatively rudimentary development. HRB has experienced high population growth in the past two decades, particularly after the Chinese reform and opening. As a result, intense competition of land for residential, industrial, agricultural, and other developmental uses is increasing in the absences of a clear urban growth policy and adequate control. The Press Conference on New Urbanization Plan of China [6] stated that the Government has supreme authority in formulating the country's urbanization policy and can promote the balanced development of geographical space to create new regional economic growth poles. Therefore, this region holds great theoretical and practical significance for studies of sustainable development in regional towns. The focus of the present study 
is reconstruction of urban extension process of this area in different time periods by using remote sensing data.

Remote sensing images contain large amounts of information of continuous space and time characteristics, which enable cyclical simulation analyses of urban expansion at different scales [7]. This approach is advantageous for extracting urban information on individual cities or large metropolises to analyze the changes in urban land. This can be accomplished by using high resolution remote sensing images $[8,9]$ such as those provided by Landsat satellite data [10], SPOT (Satellite Pour l'Observation de la Terre) satellite data [11], and ZY-3 (Ziyuan-3) satellite data [12], as well as radar data $[13,14]$. High precision information for urban extraction can be achieved by merging several data sets $[15,16]$. However, owing to the large amounts of imagery data required, studies can be constrained by the high costs of obtaining coverage; thus, small coverage analyses with monoimages are often used to extract information on single cities [17]. Remote sensing images with low resolution can also be used, which are derived from DMSP/OLS (Defense Meteorological Satellite Program/Operational Linescan System) data and MODIS (Moderate Resolution Imaging Spectroradiometer) data; such data have been used to generate static city global maps [18]. In the early 1970s, researchers developed a linear scanning business system known as the OLS of the United States DMSP that can capture urban lighting data at night in cloudless cases; such data were found to be useful for studies of urbanization [19]. Currently, DMSP/OLS data are widely used by scholars at home and abroad as effective data sources for monitoring human activities and researching urbanization [20-25].

Two types of methods are used to extract urban information from DMSP/OLS data. The first category is selected for image segmentation according to the area threshold, and the second is characterized by image classification, which uses pixels as the basic unit. The latter includes mainly classification methods that employ support vector machines (SVMs) [26] and the method of linear decomposition of mixed pixels [27]. The first method is complex and timeconsuming at both the regional and global scales, particularly when applied in research areas showing development differences among towns [28]. Compared with the threshold dichotomy, the method of image classification circumvents the threshold determination, which converts the process of urban information extraction into a classification problem whereby urban information is distinguished from noncity related information. Combining SPOT-vegetation (VGT) data, which reflect the amount of vegetation cover, with DMSP/OLS data can improve the accuracy of information extraction in urban areas. Some studies have demonstrated that remote sensing data can be used with multiple sources and low resolution for research involving urban information extraction [29, 30]. Hence, for research of large areas and long time series of the urban expansion process, the extent of urban and make maps for spatial analysis can be extracted on the basis of DMSP/OLS data and SPOT-VGT data along with the SVM classification method [31, 32].

OLS sensors neither perform data correction nor consider atmospheric changes. Interference from clouds and pollution must be considered as influencing factors of the continuity and truth of DMSP/OLS and SPOT-VGT data sets during collection [33], which limits the applications of the extracted urban information for the two types of data.

The present study has three main objectives. First, on the basis of the mutual correction of the light data and reconstruction of the quality of the Normalized Difference Vegetation Index (NDVI) derived from SPOT-VGT data, this study aims to extract urban spatial information in the experimental area by using SVM methods. Second, an accurate reconstruction of the spatial and temporal patterns of urban expansion in the HRB is attempted by using remote sensing data. Finally, on the basis of the reconstruction of the sequences of time and space involved in the process of urban expansion, this study discusses the urban expansion process detected in the HRB, thereby providing technical support and decision-making references for regional urban planning and development efforts.

\section{Study Area}

The HRB is located in the eastern part of China between the Yangtze River and the Yellow River basin and spans Henan, Hubei, Anhui, Jiangsu, and Shandong provinces with a total area of $2.7 \times 105 \mathrm{~km}^{2}$. The HRB connects the east and midwest regions of China and is the central economic zone between the Yangtze River Economic Zone and the Yellow River Economic Zone. These regions have multiple transitivity on nature and society including transitions in climate, agriculture, and culture. The HRB population is 170 million, accounting for $12.3 \%$ of the Chinese population. This high population density has an average of 611 people per square kilometer, which is 4.8 times the average Chinese population density [34]. Because the HRB is a natural geographic unit, it has not overlapped with various administrative unit boundaries. Therefore, this study includes only 33 urban areas of the $\mathrm{HRB}$, which have complete administrative units (Figure 1).

\section{Data and Methods}

3.1. Data Processing Flow. In this paper, the HRB urban location information for 1998, 2001, 2004, 2007, 2010, and 2013 was extracted by using multisource remote sensing data, and expansion of the urban system was analyzed. The specific process employed is shown in Figure 2.

First, multiple sensor DMSP/OLS and SPOT-VGT data were used for mutual correction and data preprocessing. Second, we used the SVM classification method to extract urban information for the experimental area. Finally, we analyzed the extraction results regarding the index features and spatial characteristics from global and various regional perspectives to obtain the expansion rules of the HRB for 1998-2013.

\subsection{Data and Preprocessing}

(1) DMSP/OLS Data. The DMSP/OLS lighting data were used for urban information extraction and were downloaded from 


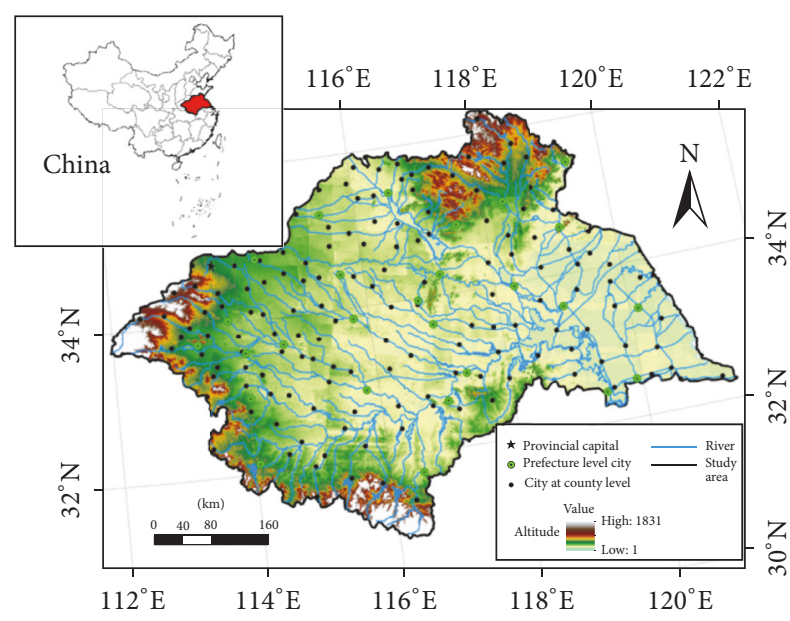

Figure 1: Study area.

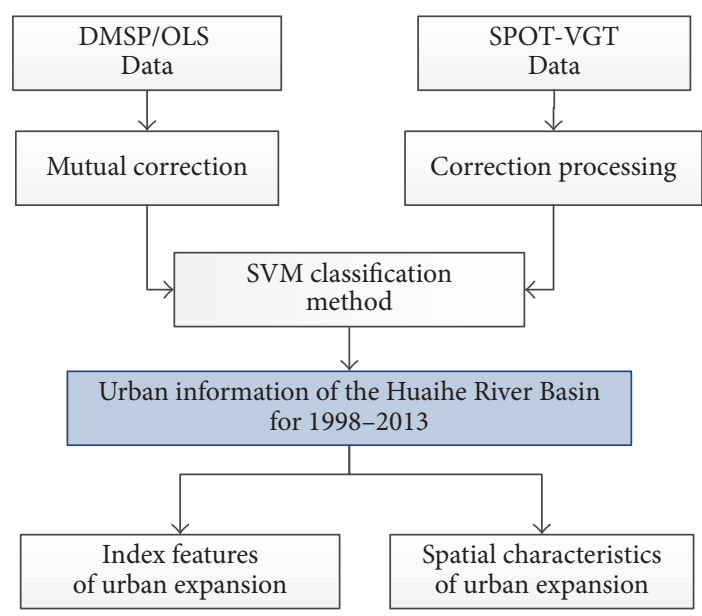

FIGURE 2: Data processing flow.

the United States National Geophysical Data Center (NGDC) [35]. To ensure that the DMSP/OLS data in the long time series have continuity and comparability, data correction was performed manually. According to the principle of accumulating high digital number (DN) values and good continuity, this study applied the data the F16 satellite recorded in 2007 for reference. Specifically, from this reference data, the lighting data at night for Jixi City were compared with our data set [24].

(2) SPOT-VGT Data. This work employed a data set of NDVI remote sensing data compiled from synthetic images that were composed over 10-day periods by the SPOT-VGT satellite; these data were obtained from the Belgium Flemish Institute for Technological Research [36]. This study used the data to improve the extraction process for urban information, and NDVI data were extracted for the long time series. Because NDVI data reflect changes in vegetation, those in the time series illustrate the rising, falling, and stable trends in vegetation in the HRB. In this study, quality reconstruction work for the NDVI data was conducted by using the Moving Weighted Harmonic Analysis (MWHA) method [33].

(3) Other Secondary Data. Auxiliary data that were used in this study included data on administrative divisions, statistical, social, and economic data [37].

3.3. Extraction Method for Urban Spatial Information. This study employed the SVM method to extract the urban information for the entire research extent during the different time periods.

The principle of SVM is that certain nonlinear mapping (kernel functions) of the input vector map is changed into a high-dimensional feature space, where it can build the optimal separation hyperplane. The algorithm establishes a decision function, and the data can be classified during testing as being the most accurate through the decision function. The decision function is calculated by formula (1) in the nonlinear case.

$$
\begin{aligned}
f(x) & =\operatorname{sign}\left(\sum_{i=1}^{n} y_{i} a_{i} K\left(x_{i}, x\right)+b\right), \\
b & =\frac{1}{N_{\mathrm{NSV}}} \sum_{x_{i} \in J N}\left(y_{i}-\sum_{x_{i} \in J} a_{j} y_{j} K\left(x_{i}, x_{i}\right)\right) .
\end{aligned}
$$

Among these terms, $K\left(x_{i}, x\right)$ is the kernel function, $N_{\mathrm{NSV}}$ is the standard supporting vector number, $J N$ is the set of the standard supporting vector, $J$ is the set of the supporting vector, and $\alpha_{i}$ is a Lagrange multiplier. The key link of the SVM algorithm is the choice of the kernel function, and the kernel function selected needs to be suitable for the Mercer's conditions [38]. In the process of classification, the pixels are divided into the urban category. The initial samples in that category are combined as training samples for the urban reclassification. This iterative process is repeated until no new pixels for urban category are present during the separation. All of the pixels for urban category at this time are the final classification results.

The DMSP/OLS and SPOT-VGT data were applied to the data merging steps as follows. First, the DMSP/OLS and SPOT-VGT data were pretreated to obtain strong continuity and consistency of the DMSP/OLS and NDVI results. The DMSP/OLS results are based on the cumulative DN value and the good continuity principle of the intercalibration data. Second, the principle for the selection of the training samples was determined. Here, the DN values greater than or equal to 40 pixels were used as urban area training samples, that is, as seeds, and DN values less than 40 and NDVI values greater than or equal to 0.4 pixels were used as nonurban area training seed samples. Finally, the two types of data were combined. We used the SVM classification method to extract the urban information.

\subsection{Analysis Method for Urban Expansion}

3.4.1. Average Annual Rate of Change in the Urban Expansion Area. The average annual change rate of the urban expansion 
area is the average growth rate of the urban area per year, as shown in formula (2).

$$
V_{T}=\frac{S_{B}-S_{A}}{T},
$$

which represents the growth rate of the urban area in the $T$ time period. $S_{A}$ and $S_{B}$ represent the urban area for times $A$ and $B$, respectively, where time $B$ follows time $A$.

3.4.2. Intensity of Urban Expansion. The Urbanization Intensification Index (UII) can be used to describe the speed and intensity of urban expansion. Large UII values are indicative of faster rates of expansion in urban areas and thus suggest that a city has assumed a large amount of nonurban space in the process of development. Conversely, small UII values are indicative of lower rates of expansion in urban areas and suggest that fewer nonurban areas have been taken.

Compared with the average annual rate of change in urban expansion, the UII introduces the regional urban area to carry out standardized treatments, which makes the expanding speed of different periods of urban area utilization more comparable; it also illustrates the relationship between urban area and nonurban area during the expansion of urban area. The formula for calculating the UII is as shown in formula (3).

$$
\mathrm{UII}=\frac{U_{i b}-U_{i a}}{T L A} \times \frac{1}{T} \times 100 .
$$

Among these terms, UII is the period of the urban expansion intensity index, $U_{i b}$ represents the end point for the urban area, $U_{i a}$ denotes the early urban area, TLA is the urban area within the study area, and $T$ is the length of the study period, where the units are in years.

3.4.3. Dynamic Degree of Urban Expansion. The annual average rate of change in urban expansion reveals the change in urban expanding area only in terms of the absolute volume. The dynamic degree of urban expansion introduces the initial urban area in the annual average rate of change, which means that the calculation results can reflect the relative dynamic changing rate of urban expansion. The formula for calculating the dynamic degree of urban expansion is shown in formula (4).

$$
K=\frac{U_{i b}-U_{i a}}{U_{i a}} \times \frac{1}{T} \times 100 \%,
$$

wherein $K$ is the dynamic degree of urban expansion in the study time period, $U_{i b}$ represents the urban area at the end, $U_{i a}$ is the initial urban area, and $T$ is the interval length of time, where the units are in years.

3.4.4. Spatial Characteristics of Urban Expansion. The spatial feature structure changes during the processes of development and expansion of a city. Gao et al. [39] divided the urban expansion in China into the following four types: (1) filling type, (2) epitaxial type, (3) enclave type, and (4) corridor type. Urban extension axes are an indispensable part of the spatial structure of urban expansion and can be divided into two types based on the angle of the extension of the urban area: (1) extension axes along land transportation lines and (2) extension axes along the river system. In the course of the actual expansion of the urban area, along the stretching axes formed two main types of urban space: centralized types of urban space and multiple grouping types of urban space.

\section{Results}

In this study, urban information for the HRB was extracted from 1998 to 2013; the results are shown in Figure 3. The urban system space-time evolution laws of the HRB over the 16 years period were analyzed from the perspective of global and regional aspects based on the extraction results.

4.1. Average Annual Change Rate for the Urban Expansion Area. The gradually increasing urban area was the most significant and intuitive feature of the urban expansion. The changes in urban area within the HRB are shown in Figure 4.

Overall, the urban area in the HRB increased between 1998 and 2013. The growth rate from 1998 to 2001 was slow, although the urban area showed a marked increase after 2001. The urban expansion increased linearly from 2001 to 2013.

As shown in Figure 4, from 1998 to 2013, the urban area's annual growth rate in the HRB did not maintain a rising trend. Specifically, the growth slowed between 1998 and 2001 and increased suddenly in 2001. Thereafter, the fast growth stage continued, and the growth rate reached $665.33 \mathrm{~km}^{2}$ per year from 2001 to 2004, which was the largest value detected. The growth slightly decreased from 2004 to 2007; afterward, the growth continued at a rate of $550-650 \mathrm{~km}^{2}$ per year. On the whole, the average annual growth rate of the HRB urban remained in a relatively stable state.

4.2. Urban Expansion Intensity. In this study, 33 urban areas were analyzed according to the urban expansion intensity, as shown in Table 1.

Table 1 shows that in most of the HRB, the UII values were minimum for 1998 to 2001 and maximum between 2007 and 2013. In Henan province, the UII values of Zhengzhou were obviously higher than those in the other cities for each period, and the index values for Xinyang were relatively low. In Anhui province, the UII reached 0.8 from 2001 to 2007 and, then, plunged to 0.05 between 2007 and 2010; the urban expansion intensity of the other regions was relatively stable. In Jiangsu province, Taizhou's UII values maintained a high trend from 1998 to 2013, and a peak of 2.05 appeared between 2010 and 2013. As a whole, the urban expansion intensity of Jiangsu province was slightly higher than that of the other three provinces from 1998 to 2013.

4.3. Dynamic Degree of Urban Expansion. According to Li et al. [40] and the situation in HRB, urban expansion in the basin was divided into five types: slow speed expansion, at $(K \leq 5)$; low speed expansion, at $(5<K \leq 10)$; medium speed expansion, at $(10<K \leq 20)$; rapid speed expansion, at $(20<K \leq 25)$; and high speed expansion, at $(K>25)$. 


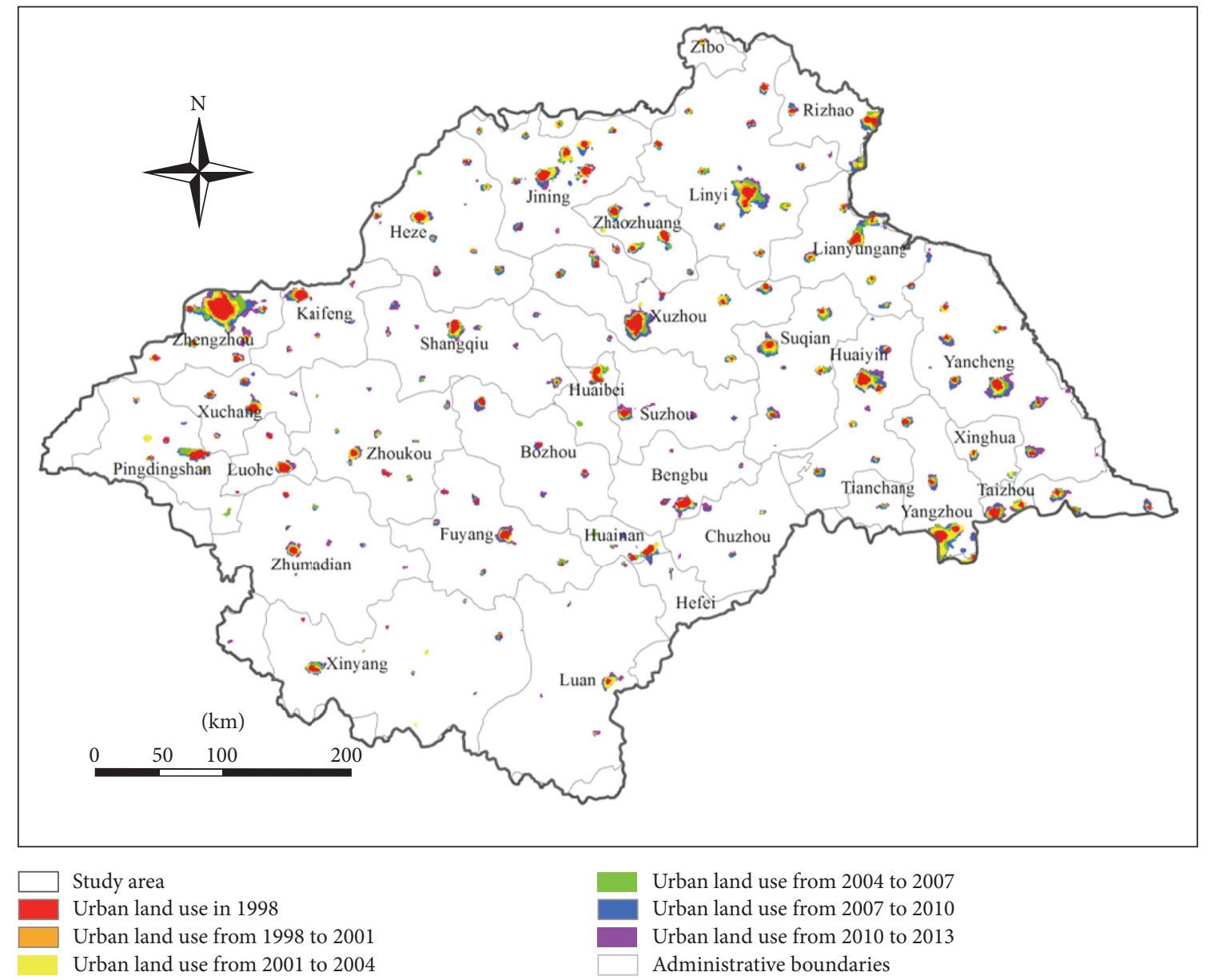

FIGURE 3: Urban information extraction results from 1998 to 2013 in HRB.

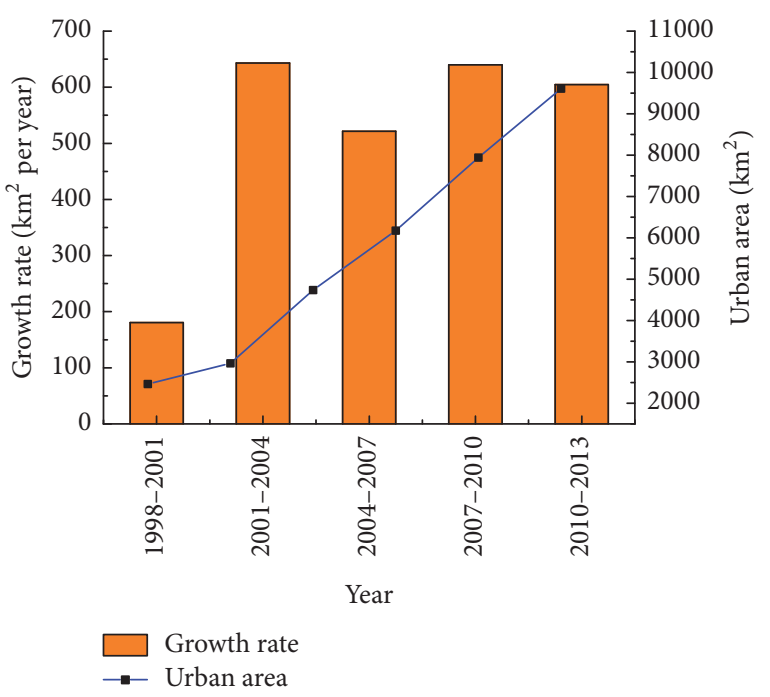

FIGURE 4: Changes in urban area between 1998 and 2013 in HRB.

As shown in Table 2, HRB's urban area was in slow expansion from 1998 to 2001, rapid expansion from 2001 to 2004, medium speed expansion from 2004 to 2007, and low speed expansion from 2007 to 2013, which may be related to reinforcement of the regional development planning regulation policy since 2000 .

The dynamic degree of expansion in each city in different periods varied significantly. Henan was in low and medium speed expansion stages from 1998 to 2013. Anhui was in low and rapid expansion stages in 1998 to 2001 and 2001-2004, respectively; afterward, medium speed expansion was maintained. Jiangsu was in low and high speed expansion stages in 1998-2001 and 2001-2004; afterward, medium speed expansion was maintained. Shandong was in a medium speed expansion stage from 1998 to 2001; afterward, slow expansion was maintained.

Although the entire HRB and each of its provinces showed low and medium speed expansion, some cities were in the high expansion stage during particular periods, such as Tianchang from 1998 to 2001; Zhumadian, Xinyang, Pingdingshan, Liuan, Huainan, Yancheng, Suqian, Huaian, Yangzhou, Xinghua, Nantong, and Zaozhuang from 2001 to 2004; Chuzhou and Tianchang from 2004 to 2007; Hefei and Chuzhou from 2007 to 2010; and Shangqiu, Zhoukou, Zhumadian, Suzhou, Liuan, Hefei, and Chuzhou from 2010 to 2013. This phenomenon indicates that the overall growth of HRB was not high, although some cities experienced a high 
TABLE 1: Urban expansion intensity index between 1998 and 2013 in HRB.

\begin{tabular}{|c|c|c|c|c|c|}
\hline & 1998-2001 & 2001-2004 & 2004-2007 & $2007-2010$ & 2010-2013 \\
\hline Henan Province & 0.06 & 0.21 & 0.16 & 0.14 & 0.29 \\
\hline Zhengzhou & 0.64 & 1.21 & 1.00 & 0.94 & 1.08 \\
\hline Kaifeng & 0.07 & 0.10 & 0.06 & 0.20 & 0.31 \\
\hline Shangqiu & 0.01 & 0.16 & 0.19 & 0.08 & 0.48 \\
\hline Zhoukou & 0.01 & 0.09 & 0.07 & 0.03 & 0.31 \\
\hline Zhumadian & 0.01 & 0.08 & 0.04 & 0.04 & 0.22 \\
\hline Xinyang & 0.01 & 0.09 & 0.06 & 0.03 & 0.07 \\
\hline Luohe & 0.12 & 0.03 & 0.11 & 0.21 & 0.53 \\
\hline Xuchang & 0.01 & 0.30 & 0.12 & 0.33 & 0.27 \\
\hline Pingdingshan & 0.06 & 0.33 & 0.25 & 0.05 & 0.09 \\
\hline Anhui Province & 0.03 & 0.11 & 0.10 & 0.13 & 0.22 \\
\hline Suzhou & 0.02 & 0.07 & 0.06 & 0.12 & 0.33 \\
\hline Huaibei & 0.18 & 0.23 & 0.22 & 0.18 & 0.15 \\
\hline Bozhou & 0.01 & 0.02 & 0.08 & 0.16 & 0.20 \\
\hline Fuyang & 0.04 & 0.10 & 0.17 & 0.13 & 0.29 \\
\hline Luan & 0.01 & 0.12 & 0.02 & 0.03 & 0.15 \\
\hline Hefei & 0.00 & 0.00 & 0.03 & 0.22 & 0.19 \\
\hline Chuzhou & 0.00 & 0.02 & 0.02 & 0.13 & 0.16 \\
\hline Bengbu & 0.07 & 0.20 & 0.07 & 0.28 & 0.29 \\
\hline Huainan & 0.08 & $\underline{0.82}$ & $\underline{0.85}$ & $\underline{0.05}$ & $\underline{0.10}$ \\
\hline Tianchang & 0.04 & 0.04 & 0.11 & 0.16 & 0.11 \\
\hline Jiangsu Province & 0.07 & 0.43 & 0.33 & 0.46 & 0.27 \\
\hline Xuzhou & 0.01 & 0.36 & 0.32 & 0.55 & 0.19 \\
\hline Lianyungang & 0.20 & 0.36 & 0.44 & 0.29 & 0.02 \\
\hline Yancheng & 0.01 & 0.28 & 0.19 & 0.46 & 0.46 \\
\hline Suqian & 0.05 & 0.36 & 0.38 & 0.24 & 0.29 \\
\hline Huaian & 0.05 & 0.38 & 0.13 & 0.67 & 0.12 \\
\hline Yangzhou & 0.11 & $\underline{1.10}$ & 0.57 & 0.59 & 0.04 \\
\hline Xinhua & 0.03 & 0.34 & 0.17 & 0.23 & 0.03 \\
\hline Taizhou & $\underline{1.01}$ & $\underline{1.70}$ & $\underline{1.35}$ & 0.85 & $\underline{2.05}$ \\
\hline Nantong & 0.04 & 0.32 & 0.53 & 0.32 & 0.90 \\
\hline Shandong Province & 0.15 & 0.31 & 0.28 & 0.38 & 0.11 \\
\hline Heze & 0.17 & 0.10 & 0.12 & 0.29 & 0.09 \\
\hline Jining & 0.05 & 0.45 & 0.17 & 0.44 & 0.12 \\
\hline Zhaozhuang & 0.09 & 0.57 & 0.49 & 0.06 & 0.10 \\
\hline Linyi & 0.16 & 0.23 & 0.39 & 0.40 & 0.14 \\
\hline Rizhao & 0.36 & 0.52 & 0.32 & 0.41 & 0.25 \\
\hline
\end{tabular}

growth rate, rapidly developing urbanization, and emerging urban regions.

4.4. Spatial Characteristics of Urban Expansion. As shown in Figure 4, the expansion of most urban areas in the HRB in 1998 to 2013 was extensive type; that is, the urban expansion occurred at the original edge of the city. In addition, new urban regions were identified around expanding cities such as Zhengzhou, Linyi, Suqian, Yancheng, and Xuzhou, which reveal the features of an enclave. In Pingdingshan and Lianyungang, urban expansion as appeared to be corridor type; that is, linear extension occurred along a particular direction, usually for traffic lines or river systems. Within the extent of the HRB, the spatial form of most expanding cities appeared to be block-shaped. In addition, the extension shaft of Lianyungang and Pingdingshan was long and extended along a particular direction with the entire city assuming a strip form. Linyi urban expansion was multidirectional and appeared to be star-like. In Huaian and Yangzhou, urban expansion in 1998 and 2001 translated into massive urban areas in 2001 and 2004. Jining had multiple independent urban areas, revealing the trend of connecting urban outer contour with each increasing urban range. 
TABLE 2: Dynamic degree of urban expansion between 1998 and 2013 in HRB.

\begin{tabular}{|c|c|c|c|c|c|}
\hline & 1998-2001 & 2001-2004 & 2004-2007 & 2007-2010 & 2010-2013 \\
\hline Henan Province & 6.70 & 17.83 & 9.15 & 6.15 & 10.95 \\
\hline Zhengzhou & 10.30 & 14.89 & 8.53 & 6.37 & 6.13 \\
\hline Kaifeng & 5.88 & 7.50 & 3.40 & 10.49 & 12.68 \\
\hline Shangqiu & 1.09 & 23.28 & 17.76 & 4.88 & 25.71 \\
\hline Zhoukou & 3.18 & 21.74 & 10.97 & 3.30 & $\underline{31.23}$ \\
\hline Zhumadian & 3.45 & $\underline{33.33}$ & 8.33 & 6.67 & 29.17 \\
\hline Xinyang & 6.86 & $\underline{40.65}$ & 12.45 & 5.07 & 8.33 \\
\hline Luohe & 4.35 & 0.86 & 3.33 & 6.06 & 12.82 \\
\hline Xuchang & 0.46 & 20.27 & 5.04 & 11.92 & 7.17 \\
\hline Pingdingshan & 7.88 & $\underline{36.77}$ & 13.29 & 2.00 & 3.30 \\
\hline Anhui Province & 7.98 & 24.29 & 12.42 & 11.76 & 15.00 \\
\hline Suzhou & 10.00 & 24.36 & 12.59 & 18.82 & $\underline{32.65}$ \\
\hline Huaibei & 8.33 & 8.57 & 6.44 & 4.44 & 3.36 \\
\hline Bozhou & 3.45 & 5.21 & 18.02 & 23.98 & 17.69 \\
\hline Fuyang & 10.00 & 18.59 & 20.99 & 9.85 & 16.76 \\
\hline Luan & 7.69 & $\underline{112.50}$ & 3.81 & 5.13 & 25.19 \\
\hline Hefei & 0.00 & 0.00 & 0.00 & $\underline{222.22}$ & $\underline{26.09}$ \\
\hline Chuzhou & 0.00 & 0.00 & 33.33 & 93.33 & 29.83 \\
\hline Bengbu & 8.16 & 18.58 & 4.21 & 14.33 & 10.68 \\
\hline Huainan & 5.75 & $\underline{48.04}$ & 20.48 & 0.75 & 1.46 \\
\hline Tianchang & $\underline{33.33}$ & 16.67 & $\underline{33.33}$ & 25.00 & 9.52 \\
\hline Jiangsu Province & 5.05 & $\underline{27.27}$ & 11.40 & 11.91 & 5.27 \\
\hline Xuzhou & 0.31 & 18.04 & 10.42 & 13.38 & 3.29 \\
\hline Lianyungang & 15.94 & 19.36 & 15.04 & 6.84 & 0.36 \\
\hline Yancheng & 1.33 & $\underline{31.80}$ & 10.63 & 20.20 & 12.45 \\
\hline Suqian & 7.78 & 42.34 & 20.04 & 7.68 & 7.75 \\
\hline Huaian & 4.02 & 26.41 & 5.01 & 22.39 & 2.31 \\
\hline Yangzhou & 4.62 & $\underline{41.44}$ & 9.54 & 7.65 & 0.38 \\
\hline Xinhua & 7.41 & $\underline{72.73}$ & 11.43 & 11.35 & 1.06 \\
\hline Taizhou & 12.94 & 15.77 & 8.52 & 4.26 & 9.11 \\
\hline Nantong & 3.33 & $\underline{26.26}$ & 24.86 & 8.41 & 19.12 \\
\hline Shandong Province & 11.85 & 18.38 & 10.63 & 10.36 & 2.92 \\
\hline Heze & 24.89 & 8.65 & 7.88 & 16.01 & 3.42 \\
\hline Jining & 2.94 & 23.97 & 5.36 & 11.70 & 2.42 \\
\hline Zhaozhuang & 5.02 & $\underline{28.97}$ & 13.38 & 1.21 & 1.90 \\
\hline Linyi & 14.84 & 14.53 & 17.36 & 11.84 & 3.06 \\
\hline Rizhao & 23.89 & 20.07 & 7.68 & 7.88 & 3.98 \\
\hline
\end{tabular}

From the perspective of a different province, the expansion degree in Zhengzhou was the most rapid in Henan province; that in Anhui province was average. The expansion degree in Xuzhou was the most rapid in Jiangsu province, and that in Jining and Linyi was higher than the expansion degree in other cities in Shandong Province.

\section{Discussions}

During the study period, built-up areas increased rapidly, and the distribution urban expansion was not balanced both temporally and spatially. For example, the value at southeast coast was higher than that in other areas and peaked in 2013. These results are in agreement with the basic trend of China's urban expansion. Considering the dynamic expansion, the overall expansion of the HRB and the provinces were low and medium speeds. Overall, in addition to that in Zhengzhou, Linyi, and Xuzhou, urban expansion between 1998 and 2013 in the rest of the urban areas of HRB was not huge. After development, the urban distribution was more dispersed; the urban density was not high, and the study area did not form a complete urban agglomeration. 
TABLE 3: Elastic coefficient of residential expansion and expanding type of city.

\begin{tabular}{lcccc}
\hline Year & Built-up area & Growth rate of nonagricultural population & Elasticity coefficient & Expansion type \\
\hline $1998-2001$ & $0.90 \%$ & $7.25 \%$ & 0.12 & Insufficient expansion \\
$2001-2004$ & $54.12 \%$ & $23.74 \%$ & 2.28 & Rapid expansion \\
$2004-2007$ & $31.25 \%$ & $12.55 \%$ & 2.49 & Rapid expansion \\
$2007-2010$ & $40.13 \%$ & $19.32 \%$ & 2.08 & Rapid expansion \\
$2010-2013$ & $9.87 \%$ & $75.61 \%$ & 0.13 & Insufficient expansion \\
\hline
\end{tabular}

The expansion of Xuzhou from 1998 to 2013 can be divided into three stages; 1998-2004 was a low speed development stage. Although the urban areas expand annually, the elasticity coefficient of the urban areas increased but still did not meet the nonagricultural population growth. 2004-2010 was a rapid development stage. The elasticity coefficient of urban growth jumped to 2.49 (Table 3), which is classified as rapid expansion. This indicates that the speed of construction land expansion was too fast in this period, which opened the national real estate market and created a relationship with economic construction. However, the uncoordinated growth of construction land and nonagricultural population led to an unreasonable land use pattern, which to some extent increased the carrying capacity of land to result in agricultural land loss. In 2010-2013, the relative relationship between the rate of urban expansion and the growth rate of the nonagricultural population was more harmonious, although it was still slightly higher than the population growth rate.

This paper analyzed the correlation of urban expansion from the aspects of population and economy. The urban nonagricultural population and the GDP (Gross Domestic Product) data characterize the urban population and economic conditions. SPSS (Statistical Product and Service Solutions) was used to analyze the correlation among builtup areas, populations, and the GDP. The results show that the correlation coefficient between the built-up areas and the GDP was 0.89 with significance over $95 \%$, which indicates that Xuzhou had a strong positive correlation between GDP and built-up areas. Population growth is an important driving force for urban expansion. An increase in urban population has strongly promoted the construction of commercial and residential buildings, the construction of public transport facilities and urban public infrastructure, and the development of urban secondary and tertiary industries, thus accelerating the expansion of built-up areas and promoting the process of urban expansion. On the contrary, the expansion of the built-up areas attracts additional labor to engage in nonagricultural activities, resulting in a corresponding increase in the number of nonagricultural population. The correlation coefficient between built-up areas and nonagricultural population in Xuzhou was 0.88 with significance more than $95 \%$; that is, the nonagricultural population in Xuzhou strongly correlated with the built-up areas. These results show that the built-up areas in Xuzhou between 1998 and 2013 correlated strongly with the nonagricultural population and the GDP, which shows that the expansion of population, economy, and built-up areas is mutually influenced and promoted. Economic development and the increase in nonagricultural population has greatly promoted the expansion of built-up area.

It should be noted that, owing to the complexity of the surface features, some nonurban pixel light noises remained in the nighttime light data. Furthermore, geometric correction was not adopted; therefore, error can be introduced in the location and false positives can be generated. Accordingly, the geometric correction of light data should be studied to improve the accuracy of information extraction in the future. The HRB includes characteristics such as a high population density and an obvious contradiction between the water supply and demand. Considering these features in disaster-prone areas, follow-up research can explore various natural factors, the relationship between economic and political factors, and the development of urban areas in the HRB to analyze the driving forces on urban expansion.

\section{Conclusions}

This study used remote sensing data and processed night light data and NDVI data in a long time series. The SVM classification method was used to extract urban information, and urban expansion rules were analyzed in the HRB. The main conclusions are given in the following points:

(1) DMSP/OLS night light data and SPOT-VGT data were selected to examine urban expansion. The SVM classification method was used for extracting urban information.

(2) The urban expansion processes between 1998 and 2013 in the HRB were reconstructed, and quantitative analysis was performed from the aspects of urban area size, expansion intensity, expansion dynamic degree, and expansion pattern.

\section{Conflicts of Interest}

The authors declare that there are no conflicts of interest regarding the publication of this paper.

\section{Acknowledgments}

This work is supported by the Human and Social Sciences of Chinese Ministry of Education (Grant no. 14YJCZH028) and the National Natural Science Foundation of China (Grant no. $41571400 \backslash 41401171 \backslash 41671405)$. 


\section{References}

[1] B. Catalán, D. Saurí, and P. Serra, "Urban sprawl in the Mediterranean?: patterns of growth and change in the Barcelona Metropolitan Region 1993-2000," Landscape and Urban Planning, vol. 85, no. 3-4, pp. 174-184, 2008.

[2] G. Mills, "Cities as agents of global change," International Journal of Climatology, vol. 27, no. 14, pp. 1849-1857, 2007.

[3] S. Angel, S. C. Sheppard, and D. Civco, The Dynamics of Global Urban Expansion, The World Bank Transportation and Urban Development Department, Washington, DC, USA, 2005.

[4] L. Tu, Z. Qin, W. Li et al., "Surface urban heat island effect and its relationship with urban expansion in Nanjing, China," Journal of Applied Remote Sensing, vol. 10, no. 2, Article ID 026037, 2016.

[5] C. L. Fang, "The urbanization and urban development in china after the reform and opening-up," Economic Geograph, vol. 29, pp. 19-25, 2009 (Chinese).

[6] China's State Council, "Chinese new urbanization plan (2014-2020)," http://www.gov.cn/zhengce/2014-03/16/content_ 2640075.htm.

[7] P. Griffiths, P. Hostert, O. Gruebner, and S. V. der Linden, "Mapping megacity growth with multi-sensor data," Remote Sensing of Environment, vol. 114, no. 2, pp. 426-439, 2010.

[8] B. S. Sokhi, N. D. Sharma, and P. S. Uttarwar, "Satellite remote sensing in urban sprawl mapping \& monitoring a case study of Delhi," Journal of the Indian Society of Remote Sensing, vol. 17, no. 3, pp. 57-69, 1989.

[9] F. M. Henderson and Z.-G. Xia, "SAR applications in human settlement detection, population estimation and urban land use pattern analysis: a status report," IEEE Transactions on Geoscience and Remote Sensing, vol. 35, no. 1, pp. 79-85, 1997.

[10] M. T. Rahman, "Detection of land use/land cover changes and urban sprawl in Al-Khobar, Saudi Arabia: an analysis of multitemporal remote sensing data," ISPRS International Journal of Geo-Information, vol. 5, no. 2, article 15, 2016.

[11] C. Weber and A. Puissant, "Urbanization pressure and modeling of urban growth: example of the Tunis Metropolitan Area," Remote Sensing of Environment, vol. 86, no. 3, pp. 341-352, 2003.

[12] H. Luo, L. Li, H. Zhu, X. Kuai, Z. Zhang, and Y. Liu, "Land cover extraction from high resolution ZY-3 satellite imagery using ontology-based method," ISPRS International Journal of Geo-Information, vol. 5, no. 3, article 31, 2016.

[13] B. Rodríguez-Cuenca, S. García-Cortés, C. Ordóñez, and M. A. Alonso, "Morphological operations to extract urban curbs in 3D MLS point clouds," ISPRS International Journal of GeoInformation, vol. 5, no. 6, p. 93, 2016.

[14] Z. Zhang, P. Tang, H. Y. Li, and Z. Feng, "Refined domain model for multisource data synergized quantitative remote sensing production system," Journal of Remote Sensing, vol. 20, no. 2, pp. 184-196, 2016 (Chinese).

[15] M. K. Jat, P. K. Garg, and D. Khare, "Monitoring and modelling of urban sprawl using remote sensing and GIS techniques," International Journal of Applied Earth Observation and Geoinformation, vol. 10, no. 1, pp. 26-43, 2008.

[16] H. Taubenböck, T. Esch, A. Felbier, M. Wiesner, A. Roth, and S. Dech, "Monitoring urbanization in mega cities from space," Remote Sensing of Environment, vol. 117, pp. 162-176, 2012.

[17] X. H. Wang, Urban Expansion in China over the Past 30 Years Detected Using DMSP/OLS Nighttime Light Data, Nanjing University, Nanjing, China, 2013 (Chinese).

[18] I. Ciesin, "CIAT Global Rural-Urban Mapping Project (GRUMP),” Alpha Version: Population grids, 2004.
[19] D. Li and X. Li, "An overview on data mining of nighttime light remote sensing," Acta Geodaetica et Cartographica Sinica, vol. 44, no. 6, pp. 591-601, 2015 (Chinese).

[20] T. A. Croft, "Nighttime images of the earth from space," Scientific American, vol. 8, pp. 86-98, 1978.

[21] M. L. Imhoff, W. T. Lawrence, D. C. Stutzer, and C. D. Elvidge, "A technique for using composite DMSP/OLS 'city lights' satellite data to map urban area," Remote Sensing of Environment, vol. 61, no. 3, pp. 361-370, 1997.

[22] C. Milesi, C. D. Elvidge, R. R. Nemani, and S. W. Running, "Assessing the environmental impacts of human settlements using satellite data," Management of Environmental Quality: An International Journal, vol. 14, no. 1, pp. 99-107, 2003.

[23] C. Y. He, J. G. Li, J. Chen et al., "the urbanization model and process in Bohai surrounding area in the 1990s by using DMSP/OLS data," Acta Geographica Sinica, vol. 60, pp. 409-417, 2005 (Chinese).

[24] Z. Liu, C. He, Q. Zhang, Q. Huang, and Y. Yang, "Extracting the dynamics of urban expansion in China using DMSP-OLS nighttime light data from 1992 to 2008," Landscape and Urban Planning, vol. 106, no. 1, pp. 62-72, 2012.

[25] B. Pandey, P. K. Joshi, and K. C. Seto, "Monitoring urbanization dynamics in india using DMSP/OLS night time lights and SPOT-VGT data," International Journal of Applied Earth Observation and Geoinformation, vol. 23, no. 1, pp. 49-61, 2013.

[26] Y. Megahed, P. Cabral, J. Silva, and M. Caetano, "Land cover mapping analysis and urban growth modelling using remote sensing techniques in greater Cairo region-Egypt," ISPRS International Journal of Geo-Information, vol. 4, no. 3, pp. 17501769, 2015.

[27] Y. Yang, C. Y. He, Y. Zhao, T. Li, and Y. Qiao, "Research on the layered threshold method for extracting urban land using the DMSP/OLS stable night time light data," Journal of Image and Graphics, vol. 16, no. 4, pp. 666-673, 2011 (Chinese).

[28] X. Cao, J. Chen, H. Imura, and O. Higashi, "A SVM-based method to extract urban areas from DMSP-OLS and SPOT VGT data," Remote Sensing of Environment, vol. 113, no. 10, pp. 2205-2209, 2009.

[29] Y. Zhou, L. Liu, Y. Lu, Z. Ma, F. Xia, and Z. Shi, "Regional scale mapping of soil organic matter using remote sensing and visible-near infrared spectroscopy," Journal of Remote Sensing, vol. 19, no. 6, pp. 998-1006, 2015 (Chinese).

[30] A. Schneider, M. A. Friedl, and C. E. Woodcock, "Mapping urban areas by fusing multiple sources of coarse resolution remotely sensed data," Photogrammetric Engineering \& Remote Sensing, vol. 4, pp. 2623-2625, 2003.

[31] D. Lu, H. Tian, G. Zhou, and H. Ge, "Regional mapping of human settlements in southeastern China with multisensor remotely sensed data," Remote Sensing of Environment, vol. 112, no. 9, pp. 3668-3679, 2008.

[32] C. D. Elvidge, D. Ziskin, K. E. Baugh et al., "A fifteen year record of global natural gas flaring derived from satellite data," Energies, vol. 2, no. 3, pp. 595-622, 2009.

[33] G. Yang, H. F. Shen, L. P. Zhang, Z. He, and X. Li, "A moving weighted harmonic analysis method for reconstructing highquality SPOT VEGETATION NDVI time-series data," IEEE Transactions on Geoscience and Remote Sensing, vol. 53, no. 11, pp. 6008-6021, 2015.

[34] The Survey and Research Group with the Central Committee of China Zhi Gong Party and The Central Committee of China Zhi Gong Party, "Research report on economic development 
and environmental protection of Huaihe River Basin," China Development, vol. 15, pp. 1-7, 2015 (Chinese).

[35] NCGIA (National Center for Geographic Information \& Analysis), https://ngdc.noaa.gov/eog/download.html.

[36] Flemish Institute for Technological Research, http://free.vgt vito.be.

[37] Geospatial Data Cloud, (Chinese) http://www.gscloud.cn.

[38] V. N. Vapnik, The Nature of Statistical Learning Theory, Springer, 1995.

[39] J. L. Gao, J. J. Chen, and X. Su, "Urban expansion and its driving mechanism in China: from three main shools' perspectives," Progress in Geography, vol. 32, pp. 743-754, 2013 (Chinese).

[40] X. W. Li, J. Y. Fang, and S. L. Piao, "The intensity and modes of urban landuse growth in Shanghai," Journal of Natural Resources, vol. 18, pp. 412-422, 2003 (Chinese). 


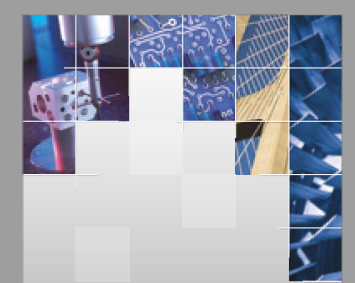

\section{Enfincering}
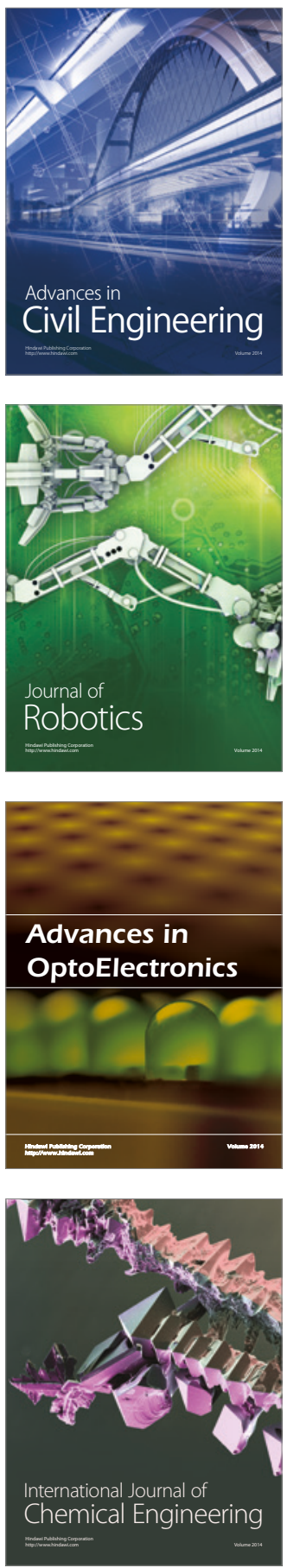

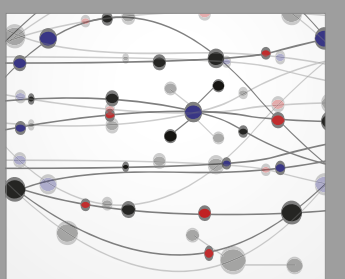

The Scientific World Journal

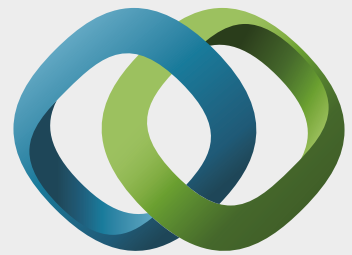

\section{Hindawi}

Submit your manuscripts at

https://www.hindawi.com
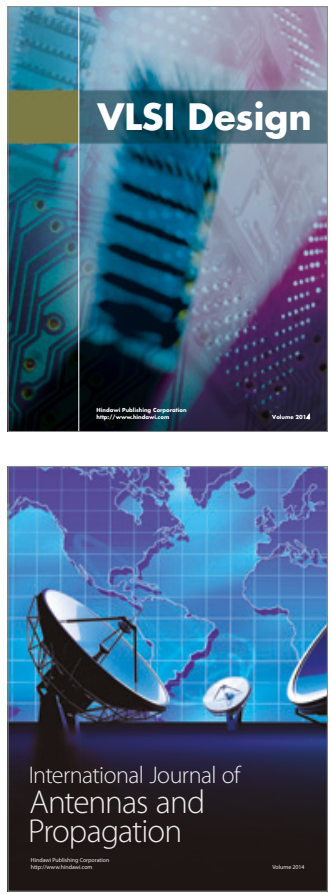

\section{Rotating}

Machinery
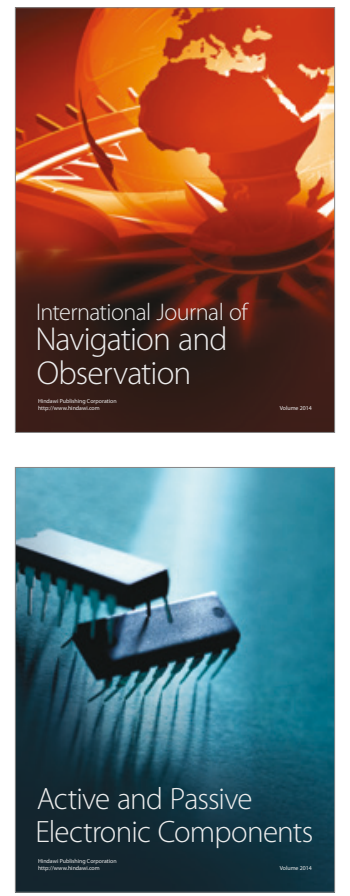
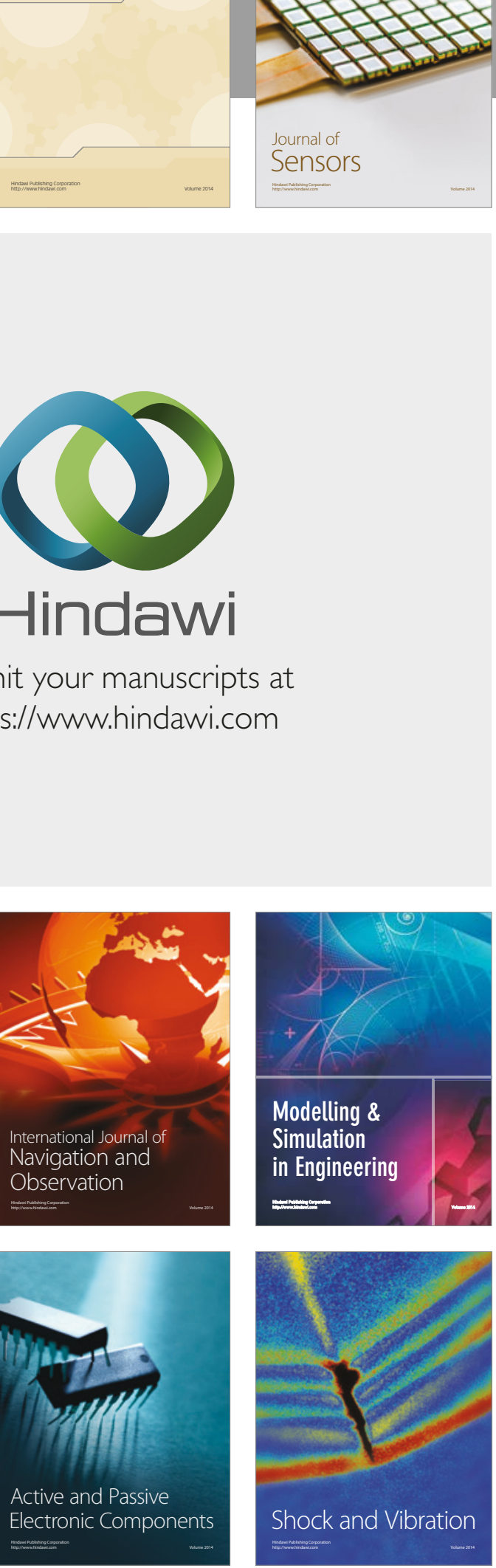
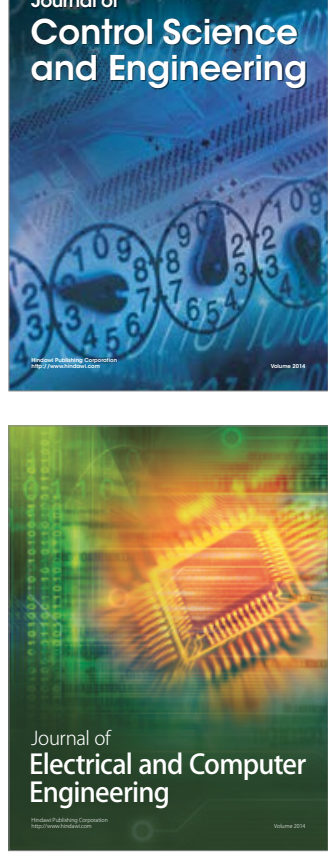

Distributed

Journal of

Control Science

and Engineering
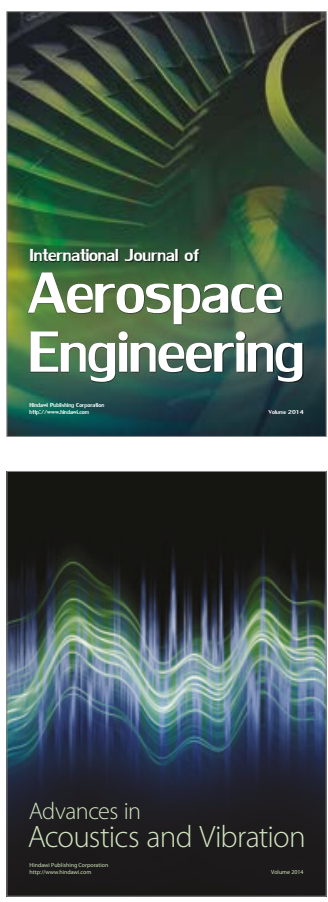

Sensor Networks 